\title{
The Cluster Head Selection Algorithm in the 3D USN
}

\author{
Pavel Abakumov ${ }^{\text {a }}$, Andrey Koucheryavy ${ }^{\text {ab }}$ \\ ${ }^{\text {a }}$ Saint-Petersburg State University of Telecommunications, Russia \\ ${ }^{\mathrm{b}}$ Central Science Research Telecommunication Institute, Russia \\ pvl.abakumov@gmail.com, akouch@mail.ru
}

\begin{abstract}
There are many cluster head selection algorithm for 2D space USN today. The LEACH algorithm is analyzed in the paper. It could be used for 3D space USN too. The new cluster head selection Maximum Coverage Algorithm (MCA) for homogeneous 3D USN is proposed in the paper. The LEACH and MCA full 3D coverage is compared by simulation on $\mathrm{C}++$. The proposed algorithm supports the full 3D space of sensor field coverage for the longer time than LEACH.
\end{abstract}

Keywords: USN, Clustering, Algorithms, Coverage.

\section{INTRODUCTION}

The Internet of Things (IoT) concept proposed by ITU-T defines one of the important directions of the telecommunication networks development [1] The Ubiquitous Sensor Networks (USN) are the technology base for IoT widely implementation [2]. The USN life-time extension and energy efficiency are the first investigation tasks during last ten years. Both of them depend from the USN cluster head selection algorithms primarily. The cluster hierarchy is the most popular USN structure today. The cluster head selection procedure should support the network life-time growth and energy efficiency in the USN.

There are many cluster head selection algorithms today. Almost all of them developed for planes. The plane algorithms which could be used for stationary USN are the LEACH (Low Energy Adaptive Cluster Hierarchy) [3, 4], HEED (Hybrid Energy-Efficient Distributed clustering) [5], for example. The plane algorithms which could be used for mobile USN are the LEACH-M [6], DCA (Distributed Clustering Algorithm) with prediction [7], for example too. The USN life-time increasing is the main investigation goal for all this algorithms.
The LEACH algorithm is the independent from space dimension and could be used for cluster head selection in 3D. The some oriented 3D algorithms were developed during recent years. The 3D space separation of the pyramids method was proposed in the $[8,9]$. The some pyramids with square shape bottoms used for sensor nodes grouping before network configuration. The main investigation goal of both papers is the USN life-time increasing too.

The Maximum Coverage Algorithm (MCA) is proposed in the paper. The main goal of MCA is the maximum coverage during long time. The sensor node coverage we define as the USN field part where this sensor supporting the reliable value of measured parameter. So, the USN coverage is the sum of these areas. The sensor node coverage for 3D space is a sphere. The simulation by $\mathrm{C}++$ was made and results were compared for LEACH and proposed algorithm. The proposed algorithm supports the full 3D space of sensor field coverage for the longer time than LEACH.

\section{NETWORK MODEL}

The network model is the homogeneous USN where the sensor node communication range $R_{\max }$ less than sensor field size. The sensor node communication range, the cluster radius and coverage zone define in accordance fig. 1.

The coverage zone or coverage radius $R_{s}$ defines as a zone where sensor supporting the reliable value of measured parameter. The coverage zone for neighboring sensor nodes can intersect. The intersection space between nodes $S_{i}$ and 
$S_{j}$ depends from the distance $d$, that can be defined by RSS (Received Signal Strength) estimation.

So:

$$
V_{S_{i} \cap S_{j}}=\frac{\pi}{12}\left(d+4 R_{s}\right)\left(2 R_{s}-d\right)^{2}
$$

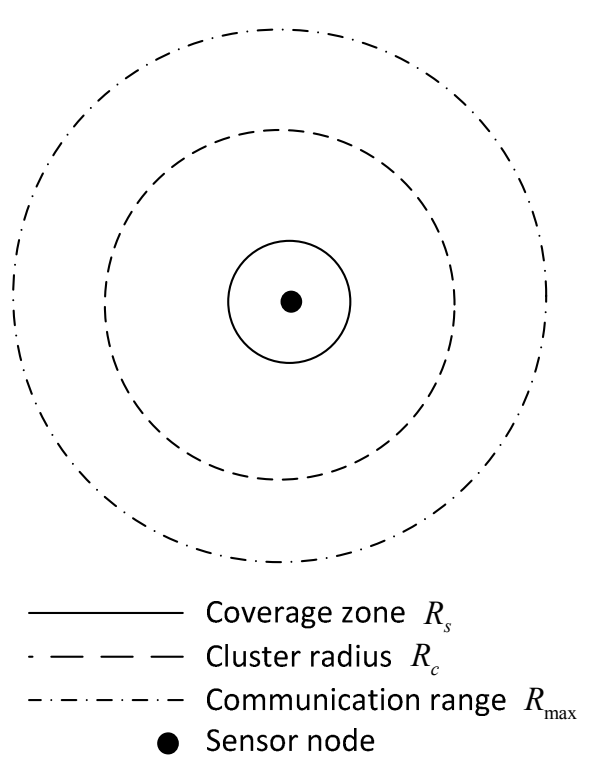

Figure 1. Communication range, cluster radius and coverage zone

The MCA algorithm assumes that each sensor node calculates the intersection space with multiplicity $U$ neighboring sensor nodes at the network initialization moment. The intersection space for i-node is the sum $V_{S_{i} \cap S_{n}}$, which divided into multiplication space coverage $V_{S}$ and number of nodes $N_{i}$. The $N_{i}$ is the number of sensor nodes that have intersection space with node $i$.

$$
V_{i}=\frac{\sum_{n \in U} V_{S_{i} \cap S_{n}}}{N_{i} V_{S}}
$$

We propose to establish a new rating parameter $W_{i}$ which presents the current status of sensor node. The USN is homogeneous and the initial sensor node energy $E_{s}$ is equal to all sensor nodes. We can use the tunable parameter $k$ which varies from 0 up to 1 . So, the rating parameter $W_{i}$ can be defined as:

$$
W_{i}=\frac{E_{i}}{E_{s}}\left(1-V_{i} * k\right)+V_{i} * k
$$

\section{Proposed Algorithm}

The USN life-time includes the network formation and the collection and transmission of information to gateway. The basic idea of proposed algorithm is the next. The network creates by neighboring sensor nodes serial poll about rating $W_{i}$ value. It should be noted that $W_{i}$ depends from the current energy balance $E_{i}$ and the space intersection $V_{i}$ value.

The sensor nodes which can be selected as cluster head node should be located in the zone $Q$. The zone $Q$ defines as a space limited by connection range $R_{\max }$ and cluster radius $R_{c}$ $\left(R_{c}<R_{\max }\right)$. The sensor node with maximum value $W_{i}$ is selected as a cluster head node. The sensor nodes which located at internal space limited by cluster radius will be the cluster members (fig.2). It's important because the size of any sensor field clusters will be similar. The selection process continues until the last cluster head node appointed. The cluster head nodes form a schedule for connection with cluster member nodes. The sensor nodes are slipping in the time period between connection sessions in according with time schedule. The network is formed.

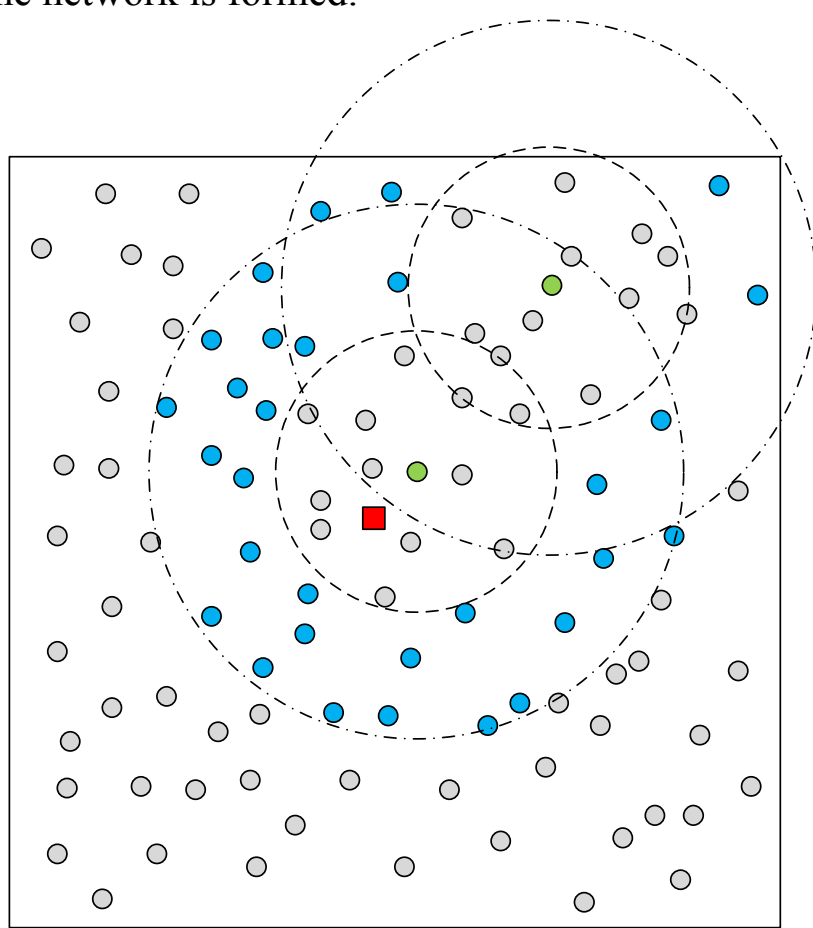

Sensor node

$\bigcirc$ Head node Base station

Candidates 
Figure 2. A network formation

Further the cluster member nodes collect and accumulate information. The cluster head nodes receive the information from cluster member nodes and transmit it to user through gateway or to gateway through another cluster head nodes. The USN formation begins from gateway neighboring clusters. So all cluster head nodes are informed about possible routing for data transmission.

\section{ALgorithm LEACH}

The Low-Energy Adaptive Clustering Hierarchy $(\mathrm{LEACH})$ algorithm is a general algorithm in the cluster head selection investigation area. There are many algorithms which based on the LEACH, for example $[10,11]$. The LEACH algorithm is the independent from space dimension and could be used for cluster head selection in 3D. The LEACH basic idea is the selection cluster head on the concrete USN history. The cluster head can be selected randomly but the sensor node which was a cluster head during previous rounds can't selection as a cluster head again. Furthermore, each sensor node generates a random number from interval $[0$, 1]. Each sensor node mapped a threshold $T_{h}(L E A C H)$ which correspond a previously defined cluster head number for USN field. The sensor node can be a cluster head if the random number for it less than $T_{h}(L E A C H)$. The threshold $T_{h}(L E A C H)$ defines as:

$$
T_{h}(L E A C H)= \begin{cases}\frac{p}{1-p \times\left(r \times \bmod \left(\frac{1}{p}\right)\right)} & \text { if } n \in G \\ 0 & \text { otherwise }\end{cases}
$$

The parameter $\mathrm{P}$ is the predefined cluster head part in the total number of USN field sensor nodes. The optimal value of $P$ is $5 \%$. The $r$ is a current round USN life-time, $G$ - the sensor nodes number which didn't selected as a cluster head during last $1 / p$ rounds. The LEACH supports the energy balance between all sensor nodes. It should be noted that the cluster head selection supplemented the control RSS node selection from cluster members.

The cluster head sends the transmission schedule to cluster members and requests the data transmission after cluster formation. The data transmission based on TDMA method. The all data collection is location at the cluster. The clusters should be formatted again after determined time. The stability USN phase is significantly more than formation phase. It's important because the LEACH overhead is definitely small.

\section{Simulation Model}

The simulation by $\mathrm{C}++$ was made and results were compared for LEACH and proposed algorithm MCA. The energy consumption for $m$ bits transmission and receiving are defined as:

$$
\begin{aligned}
E_{t x} & =E_{\text {elec }} * m+E_{\text {amp }} * m * d^{2} \\
E_{r x} & =E_{\text {elec }} * m
\end{aligned}
$$

The sensor nodes located on the USN field randomly. The gateway located in the 3D space center. The network is homogeneous and all sensors characteristics are equal. The sensor node don't participate in the USN operation in the current round if the distance to nearest cluster head is more than the communication range $R_{\max }$. It can be use in USN operation in the next round.

TABLE 1. INITIAL DATA

\begin{tabular}{|l|l|l|}
\hline Symbol & Value & Dimension \\
\hline$N$ & 2000 & number \\
\hline$P$ & 5 & $\%$ \\
\hline $\mathrm{x}$ & 100 & $\mathrm{~m}$ \\
\hline $\mathrm{z}$ & 100 & $\mathrm{~m}$ \\
\hline$E_{s}$ & 100 & $\mathrm{~m}$ \\
\hline$E_{\text {elec }}$ & 1 & $\mathrm{~J}$ \\
\hline$E_{\text {amp }}$ & 50 & $\mathrm{~nJ}$ \\
\hline$m$ & 10 & $\mathrm{pJ} / \mathrm{bit} / \mathrm{M}^{2}$ \\
\hline$R_{c}$ & 640 & $\mathrm{bit}$ \\
\hline$R_{\max }$ & 10 & $\mathrm{~m}$ \\
\hline
\end{tabular}

\section{Simulation Results}

The proposed MCA algorithm should support the USN where the sensor field can more than communication range for separate sensor nodes. The main MCA development goal is the maximum 
coverage during long time achievement for such USN's. The basic parameters for comparison with LEACH we selected coverage part and number of connected nodes during current round.

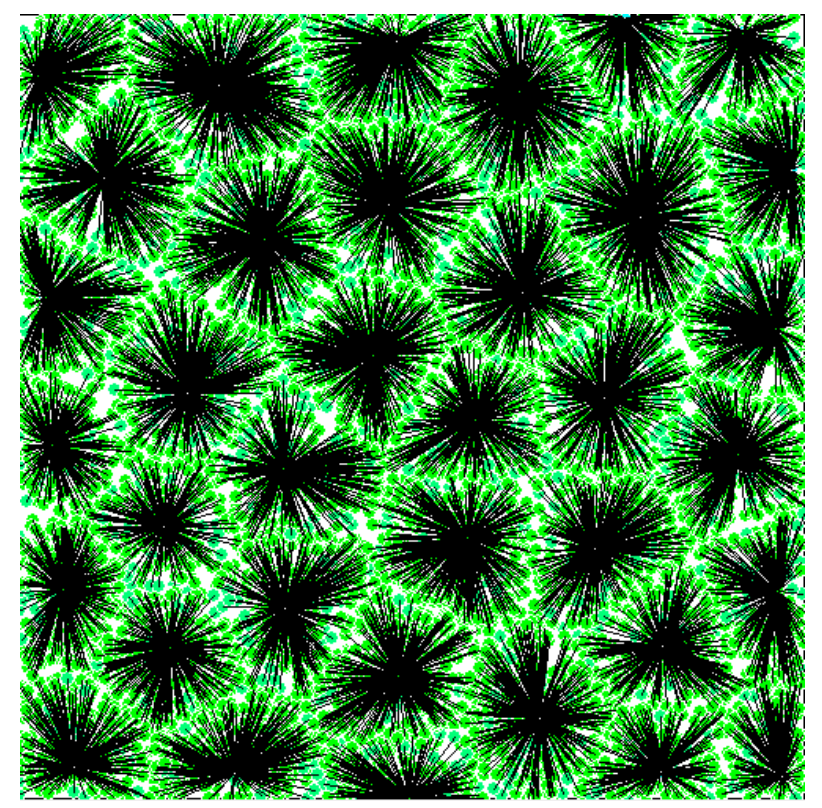

Figure 3. Sensor field clusterization

The cluster head random selection forms the clusters with difference size. Sometimes it can create the big distance between cluster heads. The several sensor nodes can't connect to any cluster head in this case. The proposed algorithm MCA can create the clusters which have the similar size (fig.3). It supports the full coverage during long time.

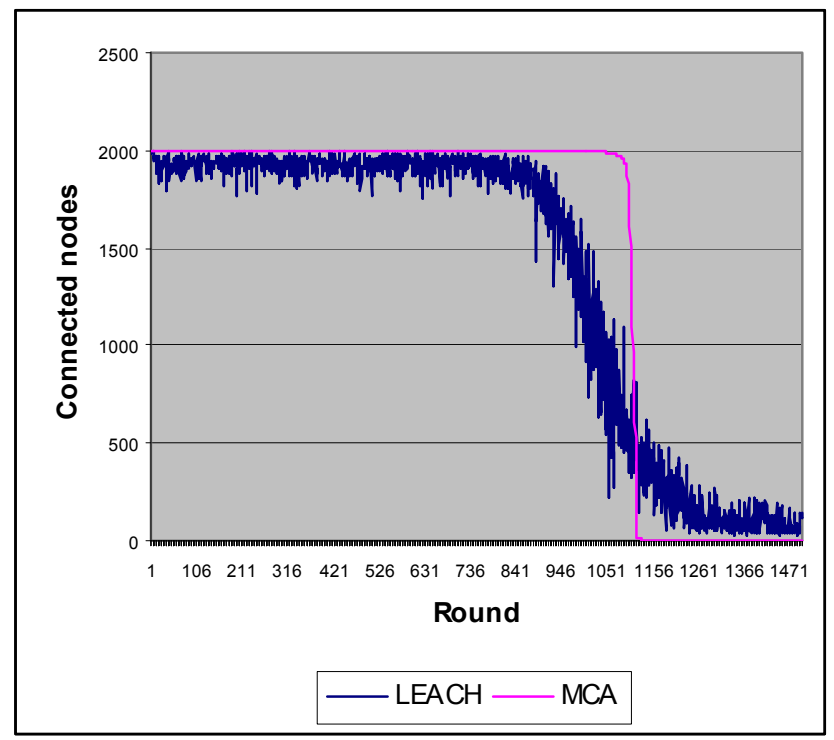

Figure 4. The number of connected nodes
The number of connected nodes as a function of the rounds is shown on the fig. 4 for LEACH and MCA algorithms. The connected nodes are the sensor nodes which participate in the USN operation in the current round. The others sensor nodes can be dead or located outside of connection range. The number of connected nodes for proposed algorithm MCA more stability than for LEACH algorithm. Furthermore, the number for connected nodes for MCA algorithm high decreases during few rounds due to the death of sensor nodes.

The sensor field coverage for LEACH and MCA algorithms is shown on the fig.5. The LEACH algorithm can't provide the $100 \%$ coverage for investigated model. It's understandable because the sensor field can more than communication range for separate sensor nodes. The proposed algorithm MCA is the significantly better than LEACH for $100 \%$ and $90 \%$ coverage. The algorithms LEACH and MCA are practically similar for less coverage rate.

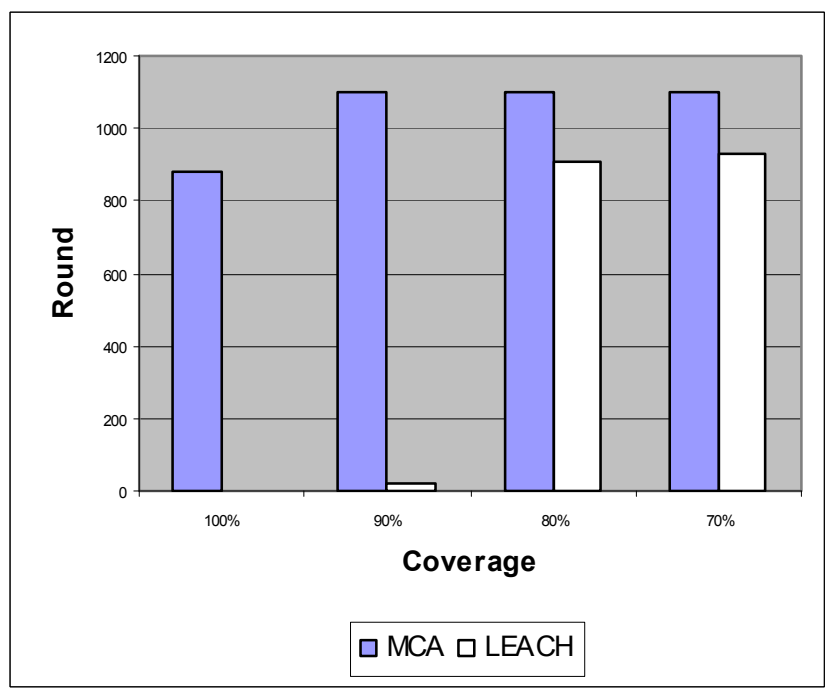

Figure 5. The coverage

\section{VII.CONCLUSIONS}

The Maximum Coverage Algorithm (MCA) for $3 \mathrm{D}$ USN is proposed in the paper. The main goal of $\mathrm{MCA}$ is the maximum coverage during long time. The simulation by $\mathrm{C}++$ was made and results were compared for LEACH and proposed algorithm. The proposed algorithm supports the full 3D space of sensor field coverage for the longer time than LEACH. The proposed algorithm MCA is the significantly better than LEACH for $100 \%$ and $90 \%$ 
coverage. The proposed algorithm can be used for the 2D USN too.

The next investigation step will be research of the big sensor fields with low sensor nodes density.

\section{REFERENCES}

[1] Recommendation Y.2060 "Overview of Internet of Things". ITU-T, Geneva. June 2012.

[2] Recommendation Y.2062. Framework of object-to-object communication using ubiquitous networking in NGN. ITU-T, Geneva. February 2012

[3] W. Heinzelman, A. Chandrakasan, H. Balakrishnan. Energy-efficient communication protocol for wireless microsensor networks Proceedings 33rd Hawaii International Conference on System Sciences (HICSS), Wailea Maui, Hawaii, USA, Jan. 2000.

[4] W. Heinzelman, A. Chandrakasan, H. Balakrishnan. An application specific protocol architecture for wireless microsensor networks. IEEE Transactions on Wireless Communications 1 (4), 2002.

[5] O. Younis, S. Fahmy. Distributed clustering in ad-hoc sensor networks: A hybrid, energy-efficient approach. Proceedings, IEEE INFOCOM, Hong Kong, China, 2004.

[6] D. Kim and Y. Chung. Self-Organization Routing Protocol Supporting Mobile Nodes for Wireless Sensor Network. Proceedings of the First International Multi-Symposiums on Computer and Computational Sciences, Volume 2, 2006

[7] A. Koucheryavy, A. Salim. Prediction-based Clustering Algorithm for Mobile Wireless Sensor Networks. Proceedings, International Conference on Advanced Communication Technology, 2010. ICACT 2010. Phoenix Park, Korea.

[8] M.Hooggar, M.Mehrani, N.Attarzadeh, M.Azimifar. An Energy Efficient Three Dimensional Cjverage Method for Wireless Sensor Networks. Journal of Academic and Applied Studies. V.3 (3), March 2013.

[9] N.Attarzadeh, M.Mehrani. A New Thre Dimensinal Clustering Method for Wireless Sensor Networks. Global journal of Cmputer Science and Technology. V.11, issue 6, version 1.0, April 2011.

[10] M.F.K.Abad, M.A.J.Jamali. Modify LEACH Algorithm for Wireless Sensor Network. International Journal Of Computer Science Issues. V.8, isuue 5, №1, September 2011.

[11] A.Koucheryavy, A.Salim, W.Osamy. Enhanced LEACH Protocol for Wireless Sensor Network. Proceedings, INTHITEN (IoT and its Enablers) conference. St. Petersburg, State University of Telecommunication, 3-4 June, 2013

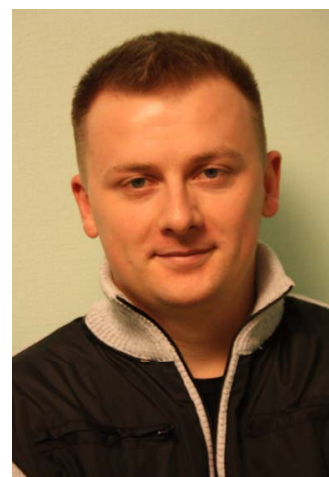

P. Abakumov was born in Leningrad 17.01.1985, graduated from Mathematics and Mechanics Faculty of SaintPetersburg State University in 2007 and received $\mathrm{M}$. Tech degree in computer science from the Bonch-Bruevich SaintPetersburg State University of Telecommunications (SUT)

$\mathrm{He}$ is Saint-Petersburg State University of Telecommunications $\mathrm{Ph}$. D. student from 2013. His current research interest include sensor networks, wireless protocol architecture and Internet of Things.

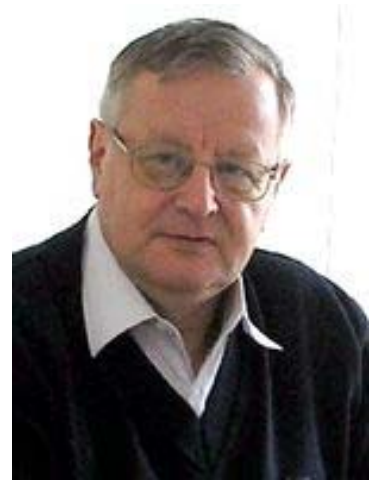

A. Koucheryavy was born in Leningrad 02.02.1952. After graduated from Leningrad University of Telecommunication in 1974 he going to Telecommunication Research Institute named LONIIS, where A.Koucheryavy working up to October 2003 ( from 1986 up to 2003 as the First Deputy Director ). He became the Ph.D and D.Sc in 1982 and 1994 respectively.

A.Koucheryavy is the St. Petersburg State University of Telecommunication (SUT) professor from 1998. He is SUT department "Telecommunication Networks" chief from 2011. Prof. A.Koucheryavy is the advisor of the Central Science Research Telecommunication Institute and St. Petersburg Branch of "GIPROSVYAZ" Institute simultaneously. He is honorary member of A.S.Popov society.

Prof. A.Koucheryavy was the vice-chairman Study Group 11 ITU-T (Study periods 2005-2008, 2009-2012 ). His scientific areas of interest are the network planning, teletraffic theory, IoT and its enablers. 